\title{
Transcriptomic dynamics changes in development of carmine radish (Raphanus sativus $L$.) fleshy roots using RNA-seq method
}

Jian Gao

Yangtze Normal University

Mao Luo

Southwest Medical University

Yi Liu

Sichuan Agricultural University

\section{Fabo Chen}

Yangtze Normal University

Hua Peng

Sichuan Tourism College

Wenbo Li ( $\nabla$ liverb@163.com )

Yangtze Normal University

\section{Research article}

Keywords: Radish (Raphanus sativus L.), Differential expression genes (DEGs), Dynamics development stage of fleshy roots, KEGG pathway enrichment

Posted Date: August 23rd, 2019

DOI: https://doi.org/10.21203/rs.2.13429/v1

License: (c) (1) This work is licensed under a Creative Commons Attribution 4.0 International License.

Read Full License 


\section{Abstract}

Radish ( Raphanus sativus L.), belonging to biennial root vegetable crop of Brassicaceae family, is an economically important vegetable crop with an edible taproot. Recently, most of differential expressed genes associating with anthocyanin biosynthesis have been identified in most of important fruit crops. However, transcriptome analysis of anthocyanin biosynthesis and expression of anthocyanin biosynthesis related genes in 'Hongxin' radish have not been fully investigated. Here, based on results from HPLC analysis, young fleshy roots obtained from the dynamics development stage of fleshy roots in carmine radish 'Hongxin 1' was used for RNA-Seq, including fleshy roots from seedling stage (SS), initial expansion (IE), full-expansion (FE), bolting stage (BS), initial flowering stage (IFS); full-bloom stage (FBS) and podding stage (PS). Subsequently, the putative candidate genes involved in the dynamics development stage of fleshy roots in carmine radish were identified. After that, DGE (differential gene expression) profile analysis was used to identify the pupative transcripts, compared with fleshy roots from seedling stage (SS). In addition, co-modulated DEGs (Common DEGs in the dynamic growing stages of fleshyroot in carmine radish) were also identified, from which most DGEs were more likely to participate in anthocyanin biosynthesis, including two transcription factors RsMYB and Rs RZFP. In addition, some related proteins e.g. RsCHS , RsDFR, RsANS , RsF'3H , RsF3GGT1 , Rs3AT1 , glutathione Stransferase F12, RsUFGT78D2-like and RsUDGT-75C1-like were significantly contributed to the regulatory mechanism during anthocyanin synthesis in the development stage of fleshy roots. Furthermore, GO terms comprised of "anthocyanin-containing compound biosynthetic process" and "anthocyanincontaining compound metabolic process" were commonly overrepresented in the other dynamics growing stages of fleshy roots after initial expansion of fleshy roots. Moreover, these results indicated that five significantly enrichment pathways of DEG were identified for the dynamics growing stages of fleshy roots in carmine radish, including Flavonoid biosynthesis, Flavone and flavonol biosynthesis, Diterpenoid biosynthesis, Anthocyanin biosynthesis, as well as Benzoxazinoid biosynthesis. These results will expand our understanding of complex molecular mechanism of the putative candidate genes involved in the dynamics development stage of fleshyroot in carmine radish.

\section{Background}

Anthocyanins, recognized as regulator for red to purple colors in nature, thereby producing water-soluble pigments belonging to the flavonoid group [1]. Most research has demonstrated that anthocyanins, as the benefit food additive worldwide, could release major public health threat (comprised of cardiovascular disease, inflammatory, obesity, and diabetes) caused by chemical-synthesis food additive[2, 3]. In addition, most of regulatory genes have been extensively found involved in the anthocyanin biosynthetic pathway, which were largely conserved among flowering plants[4]. Previous studies have demonstrated that anthocyanins are firstly formed from phenylalanine by series enzymes through phenylpropanoid metabolism, such as phenylalanine ammonia-layse (PAL), cinnamic 4-hydroxylase (C4H) and 4coumarate-CoA ligase (4CL); ; subsequently followed by chalcone synthase (CHS),, and then the product 4 , 2' 4' 6'-tetrahydrocychalcone are further catalyzed successively by four enzymes [Chalcone Isomerase 
$(C H I)$,, flavanone 3-Hydroxylase $(F 3 H)$,, dihydroflavonol 4-Reductase (DFR),, as well as anthocyanidin synthase $(A N S / L D O X)][[5,6]$. However, the molecular mechani $m$ of anthocyanin biosynthesis regulation is still not fully understood in the dynamic development of fleshy roots in radish.

Recently, based on the global transcriptome technology (such as RNA-seq technology), most of differential expressed genes associating with anthocyanin biosynthesis and expression of anthocyanin biosynthesis have been identified in most of important fruit crops. However, transcriptome analysis of anthocyanin biosynthesis and expression of anthocyanin biosynthesis related genes in 'Hongxin' radish (That is famous for containing natural red pigment (red radish pigment), as produced in Chongqing Fuling named for Fuling three specialties) have not been fully investigated.

\section{Results}

\section{Dynamics Anthocyanidin profiles of fleshy root in development of carmine radish}

To demonstrate the dynamics Anthocyanidin profiles of fleshy root in development of carmine radish, Anthocyanidin profiles of fleshy root from seedling stage, initial expansion,full-expansion,Bolting stage, initial flowering stage,full-bloom stage and podding stage in five local cultivars of carmine radish ('Hongxin 1', 'Guanguan,'Longquan 1,' Yanzhi 1' and 'Yanzhi 2') were investigated by HPLC analysis. The results showed that Anthocyanidin was significantly increased in 'Hongxin 1'differential fleshy roots types with the development of dynamic growing stages of fleshy roots than other local cultivars of carmine radish, from seedling stage to full-bloom stage, but decresed in podding stage (Fig. S1).

\section{Illumina sequencing and De novo assembly}

Based on results from HPLC analysis, young fleshy roots obtained from the development stage of carmine radish 'Hongxin 1' was used for RNA-Seq in this study. The cDNAs obtained from fleshy roots of seven growth phases (seedling stage, initial expansion, full-expansion, Bolting stage, initial flowering stage, full-bloom stage and podding stage) were sequenced using Illumina sequencing technology. After filtering out adaptor-only reads, trimming reads and low-quality reads (base quality $\leq 10$ ), high-quality Reads were aligned to the SSU and LSU rRNA sequences to remove rRNA reads by a home-made perl script. And the percentage of clean reads counts almost $70 \%$ after removing rRNA sequences on average among raw tags in each library, respectively (Table 1). Moreover, 198, 342 assembled transcipts from the raw sequence reads were constructed with an average length of $411 \mathrm{bp}$, and 34,927 Unigenes were generated using paired-end reads with an average length of $768 \mathrm{bp}$ through de novo assembly technology (Fig. S2). 


\section{DEGs related to the dynamics growing stages of fleshy roots in carmine radish}

To identify the putative candidate genes with great changes involved in the dynamics growing stages of fleshy roots in carmine radish, normalized expression levels for all global expressed genes were analyzed, indicating that high distinct gene expression profiles exists in the dynamics growing stages of fleshy roots (Fig. 1, Table S2).. More interestingly, we found that the putative candidate genes belong to Cluster 8 was consistently with dynamics anthocyanidin profiles of fleshy root in development of carmine radish, but the putative candidate genes categorized into Cluster 9 were found oppositely.

Furthermore, DEGs were identified the dynamics growing stages of fleshy roots among other different development periods (IE_root', 'FE_root', 'BS_root', 'IFS_root', 'FBS_root' and 'PS_root) between 'SS_root' group, the results indicated that 1,629, 1,037, 1,385, 1,521, 1,574 and 917 DEGs were generated in IE_root', 'FE_root', 'BS_root', 'IFS_root', 'FBS_root' and 'PS_root', compared with 'SS_root', including up-regulated $(878,755,718,838,852$ and 555 transcripts) and down-regulated genes $(751,282,667,683,722$ and 362 transcripts) (Fig. 2A).. 126 Co-modulated DEGs (Common DEGs in the dynamic growing stages of fleshy roots in carmine radish) were identified based on venny graph (Fig. 2B),, and expression changes pattern of co-modulated DEGs were displayed with different colors using heatmap (Fig. 2C).. More importantly, we found some of co-modulated DEGs showed similar expression trends in the dynamics growing stages of fleshy roots, which was found consistently with anthocyanidin profiles of fleshy root in development of carmine radish, such as series functional enzymes acted as important regulators in anthocyanins biosynthesis, including dihydroflavonol 4-reductase (DFR: Cluster_13775), flavonoid 3'-monooxygenase (F3'H: Cluster_4431), leucoanthocyanidin dioxygenase (ANS: Cluster_3903) and Chalcone synthase (CHS: Cluster_39833), as well as some regulation enzymes comprised of anthocyanidin 3-0-glucoside 2"-0xylosyltransferase(F3GGT1: Cluster_9270), coumaroyl-CoA:anthocyanidin 3-0-glucoside-6"-0coumaroyltransferase 1-like (3AT2: Cluster_46827), UDP-glycosyltransferase 75C1-like (UGT75C1: Cluster_2736) and UDP-glycosyltransferase 78D2-like (UGT78D2: Cluster_11854). In addition, sets of transport proteins and transcription factors, such as, glutathione S-transferase F12 (Cluster_24268), MYB transcription factor (Cluster_28373), as well as Zinc finger, RING-type protein (Cluster_7186) (Fig. 2C, Table S3). Moreover, these DEGs related to the dynamics growing stages of fleshy roots that involved in different biological processes were validated using qRT-PCR and the results showed higher consistent with expression profiles of RNASeq data (Fig. 3, Table S4)..

\section{Functional annotation of DEGs related to the dynamics growing stages of fleshy roots in carmine radish}

To explore the regulatory mechanisms of DEGs related to the dynamics growing stages of fleshy roots in carmine radish, GO annotation and KEGG pathway enrichment of those putative DEGs were conducted. The results illustrated that GO terms comprised of "anthocyanin-containing compound biosynthetic 
process" and "anthocyanin-containing compound metabolic process" were commonly overrepresented in the other dynamics growing stages of fleshy roots after initial expansion of fleshy roots (IE-40 days after planting), "flavoriod biosynthetic process" and "flavoriod metabolic process" were found overrepresented in fleshy roots of IFS, FBS and PS; but for "pigment biosynthetic process" and "pigment metabolic process", which was only found overrepresented in fleshy roots of IFS and FBS; moreover, we found that GO terms comprised of "glucosinolate biosynthetic process" and "glucosinolate metabolic process" were only overrepresented in fleshy roots of IE. (Fig. 4A, Table S5).. By conducting pathway enrichment analysis, these results indicated that five significantly enrichment pathways DEG were identified for the dynamics growing stages of fleshy roots in carmine radish, including Flavonoid biosynthesis, Flavone and flavonol biosynthesis, Diterpenoid biosynthesis, Anthocyanin biosynthesis, as well as Benzoxazinoid biosynthesis (Fig. 4B, Table S6)..

\section{Discussion}

Anthocyanins have reported as important regulators for many of the red, purple and blue pigments in plants as a subgroup of flavonoids. Based on the anthocyanidin structures cyanidin, delphinidin and pelargonidin, more than 550 different anthocyanins have been isolated from diverse plant and identified by the extent of hydroxylation in the flavonoid B ring [12]. The researches have demonstrated that anthocyanin compounds were synthesized from methylation, glycosylation and acylation of the basic flavonol structure. Several structural genes and enzymes have been reported involved in anthocyanin and flavonoid biosynthetic pathway in fruits of most of plant species. Previous studies have demonstrated that anthocyanins are firstly formed from phenylalanine by series enzymes through phenylpropanoid metabolism, such as Chalcone synthase $(\mathrm{CHS})$ contributed in flavonoid synthesis as the first important enzyme, followed by Chalcone isomerase ( $\mathrm{CHI}$ ) that acted as a central branching point of the flavonoid pathway, thereby leading to the formation of flavanones through closing the C-ring[13]. After that, Dihydroflavonol 4-reductase (DFR) and anthocyanidin synthase (ANS) were used to create proanthocyanindins for formation of anthocyanidins, or anthocyanins through the action of UDPflavonoid glycosyltransferases (UFGTs)[14]. Here, dihydroflavonol 4-reductase (DFR: Cluster_13775), flavonoid 3'-monooxygenase (F3'H: Cluster_4431), leucoanthocyanidin dioxygenase (ANS: Cluster_3903) and Chalcone synthase (CHS: Cluster_39833) were identified and validated using qRT-PCR, which was significantly up-regulated in the dynamics growing stages of fleshy roots among other different development periods from seedling stage to full-bloom stage, but decresed in podding stage; and showed consistently with dynamics anthocyanidin profiles of fleshy root in development of carmine radish. In addition, the expression of UFGT genes has also been identified as important regulator involved in the anthocyanin biosynthetic pathway $[15,16]$ that attached sugar moieties to the anthocyanin aglycone for stabilising the anthocyanidin. In this study, UDP-glycosyltransferase 75C1-like (UGT75C1: Cluster_2736) and UDP-glycosyltransferase 78D2-like (UGT78D2: Cluster_11854) were significantly up-regulated in differential fleshy roots types with the development of dynamic growing stages of fleshy roots from seedling stage to full-bloom stage, but decresed in podding stage. After that, further modifications comprised of glycosylation, acylation or methylation existed for anthocyanins modifications, such as the 
formation of anthocyanin through a reaction catalysed by a cyanidin 3-0-glycosyltransferase. Here, anthocyanidin 3-0-glucoside 2"-0-xylosyltransferase (F3GGT1: Cluster_9270), coumaroylCoA:anthocyanidin 3-0-glucoside-6"-0-coumaroyltransferase 1-like (3AT2: cluster_9270) were demonstrated as key regulation enzyme for formation of anthocyanin in carmine radish. In addition, sets of transport proteins and transcription factors, such as, glutathione S-transferase F12 (Cluster_24268), MYB transcription factor (Cluster_28373), as well as Zinc finger, RING-type protein (Cluster_7186). To date, GSTs were found involved in anthocyanin transport based on genetic and biochemical evidence[17]. $B z 2$, firstly demonstrated in Zea mays by its mutant bronze-2 as a GST-encoding gene, which was found involved in vacuolar transfer of anthocyanins (bz2)[18]. Anthocyanin accumulation and pigment mislocalization were found reduced in Arabidopsis, caused by Mutations in the GST-encoding genes[19]. In this study, GSTs F12 was significantly up-regulated in differential fleshy roots types with the development of dynamic growing stages of fleshy roots. These findings provided further evidences for the role of GSH in anthocyanin transport mechanisms. Moreover, MYB is a key component of the central regulatory to determine variation of anthocyanin production [20]. More importantly, MYB transcription factors have been extensively studied for their roles in the regulation of pigmentation in plants. It is known that R2R3-type MYB proteins and the MYB-bHLH-WD40 complex directly activate the transcription of structural genes in the anthocyanin pathway, such as transcription of the early $\left(\mathrm{CHS}, \mathrm{CHI}, \mathrm{F}^{\prime} \mathrm{H}\right.$ and $F L S$ ) and late (DFR, ANS and ANR) flavonoid biosynthesis genes, respectively[21]. In this study, we also demonstrated that $M Y B$ transcription factors was significantly dynamically up-regulated and showed remarkable positive and significant correlation to red pigment content in differential fleshyroots types (Table S3). So we inferred that MYB transcription factors might specifically activate early flavonoid biosynthesis genes comprise of $C H S, C H I$ and $F 3^{\prime} H$, as well as late flavonoid biosynthesis genes consisted of $D F R$ and $A N S$ in carmine radish, thereby directly playing important roles in anthocyanin biosynthesis. However, their molecular regulation mechanism awaits further investigation.

\section{Methods}

\section{Plant material and experiment design}

Five local cultivars of carmine radish ('Hongxin 1', 'Guanguan','Longquan 1',' Yanzhi 1' and 'Yanzhi 2') collected from Fuling were selected as experiment materials with containing natural red pigment (red radish pigment). For identify the pigment contents of five local cultivars of carmine radish, dynamics Anthocyanidin profiles of fleshy root in development of carmine radish were investigated by HPLC analysis, including fleshy root from seedling stage (SS-15 days after planting); initial expansion of fleshy roots (IE-40 days after planting); full-expansion of fleshy roots (FE-70 days after planting); bolting stage (BS-120 days after planting); initial flowering stage区lFS-140 days after planting); full-bloom stage (FBS-160 days after planting); podding stage (PS-200 days after planting). Briefly, fleshy roots were collected from three homozygous of five local cultivars of carmine radish individuals and pooled together, respectively. The fleshy root tissues were grinded with liquid nitrogen and then we extracted the red pigment with a solvent mixture containing methanol $(40 \%, \mathrm{v} / \mathrm{v})$, formic acid $(0.1 \%, \mathrm{v} / \mathrm{v})$ and acetone $(40 \%$, 
v/v). We used a $10 \mu \mathrm{L}$ injection volume for a VDS C-18 column $(4.6 \times 250 \mathrm{~mm}, 5 \mu \mathrm{m}$, VDS Optilab, Germany) with $0.8 \mathrm{~mL}$ min-1 flow rate. Based on results from HPLC analysis, young fleshy roots obtained from the development stage of carmine radish 'Hongxin 1' was used for RNA-Seq in this study (Fig. S1)..

The carmine radish 'Hongxin 1' was cultivated in a greenhouse at the experimental farm of the Yihe (Yangtze normal university experiment base) in 2018. Firstly, we sowed seeds of 'Hongxin 1' in sterilized soil for 2 weeks under normal growth conditions $\left(23^{\circ} \mathrm{C}, 16 \mathrm{~h}\right.$ light/ $8 \mathrm{~h}$ dark). After that, 2-week-old plants were transferred and kept for 15 days in the cold room $\left(5 \pm 1^{\circ} \mathrm{C}, 12 \mathrm{~h}\right.$ light/ $12 \mathrm{~h}$ dark) for vernalization treatment. After the vernalization periods, the plants were grown in a normal growth room under normal growth conditions $\left(23^{\circ} \mathrm{C}, 16 \mathrm{~h}\right.$ light/ $8 \mathrm{~h}$ dark). At least three independent biological replicates for fleshy roots obtained from the development stage of carmine radish 'Hongxin 1' were collected for RNA-Seq with two replicates. All harvested tissue were immediately frozen in liquid nitrogen and stored at $-80^{\circ} \mathrm{C}$ for RNA-seq analysis respectively.

\section{Sample preparation and library construction}

Library construction was conducted following NEBNext Ultra RNA Library Prep Kits for Illumina (NEB, USA), the mRNA was isolated through magnetic beads with Oligo (dT) using approximately $5 \mu \mathrm{g}$ of total RNA and subsequently converted into short fragments by fragmentation buffer. After that, short fragments were converted into the first strands of CDNA used as templates with random hexamers, as well as the second strands of cDNA were also synthesized, and then the desired synthesized cDNA fragments were purified (QiaQuick PCR kit) for PCR amplification, and the quantify and qualify of each sample library were checked by agilent 2100 Bioanaylzer and ABI StepOnePlus Real-Time PCR System. Ultimately, 200bp paired-end reads were generated from the prepared library with 2 replicates using Illumina HiSeqTM 2000.

\section{Reads processing and differentially expressed genes (DEGs) identification}

Using Trimmomatic software, clean reads were obtained through filtering out adaptor-only reads, trimming reads and low-quality reads (base quality $\leq 10$ ). After that, high-quality Reads were aligned to the SSU and LSU rRNA sequences download from sillva database using bwa with paramers "-n 4 -o 1 -e 1 -i 0 -I 50 -k 2" and the mapped reads were removed by a home-made perl script as rRNA reads. Subsequently, we used Trinity software to de novo assembled clean reads into transcripts, and all their responding unigenes were annotated through searching in national center for biotechnology information (NCBI) non-redundant protein (Nr) databases and Swiss-Prot protein databases using BLASTx search tool with threshold E-value set as less than 10. After that, the functions of assembled unigenes were annotated through gene ontology (GO, http://www.geneontology.org/) database and kyoto encyclopedia 
of genes and genomes (KEGG, http://www.kegg.jp/) database. To assess the abundances of assembled transcript, we firstly mapped the clean reads of seven different fleshy roots libraries to the de novo assembled transcriptome using Bowtie2, and then assessed with RSEM through transcript quantification of the de novo assembly, only transcripts (FPKM $\geq 1$ ) were considered as significant expressed transcripts[7]. At last, DEGs (Differential expressed genes) were then screened by noiseqbio [8] and then identify using a corrected P-value $<0.05$ between each set of compared samples. (The fold change of gene expression of six cultivars of radish comprised of 'IE_root', 'FE_root', 'BS_root', 'IFS_root', 'FBS_root' and 'PS_root ' were identified by comparing with 'SS_root' respectively, including 'IE_root' Vs 'SS_root', 'FE_root' Vs 'SS_root', 'BS_root' Vs 'SS_root', 'IFS_root' Vs 'SS_root', 'FBS_root' Vs 'SS_root' and 'PS_root' Vs 'SS_root'). Furthermore, DEGs in the dynamic growing stages of carmine radish were analyzed and plotted using Neighbor-Joining cluster through homemade R script.

\section{GO functional annotation and KEGG pathway analysis of co- modulated differently expressed genes (DEGs) in growing stages of carmine radish}

Co-modulated DEGs (Common DEGs in the dynamic growing stages of fleshy roots in carmine radish) were identified based on venny graph. Subsequently, we conducted those co-modulated DEGs for GO annotation through Gene Ontology Database (http://www.geneontology.org/) and KEGG pathway enrichment analysis using KOBAS software, respectively [9]. In addition, the degree of KEGG enrichment was evaluated as the rich factor, q-value, and the number of genes in the enriched pathway. The rich factor refers to the ratio of the number of DEGs to the number of total annotated genes in a certain pathway. The q-value is a multiple hypothesis-corrected $P$ value. The q-value can take on values between 0 and 1 ; values closer to 0 indicate more significant enrichment. After that, $R$ script was used to construct their relative graphs.

\section{Validated of candidate DEGs invovled in the growing stages of carmine radish using real-time qRT-PCR}

To confirm the results obtained from the RNA-Seq assay, 11 DEGs with great alteration that related to growing stages of carmine radish were chosen and validated by qRT-PCR. The primers are designed by Primer 5.0 software for qRT-PCR experiments and radish gene (Actin) is used as a standard control (Table S1).. The amplification programs were performed according to the standard protocol of the ABI7500 system, and conducted in triplicate as mentioned by Jian et al.[10]. The relative quantitative method (2$\triangle \triangle \mathrm{CT}$ ) was used to calculate the fold change in the expression levels of target genes[11].

\section{Abbreviations}


ASRGs: Anthocyanin synthesis-related genes; PAL: Phenylalanine ammonia-layse; $\mathrm{C} 4 \mathrm{H}$ : Cinnamate 4hydroxylase; 4CL: 4-coumarate: $\mathrm{CoA}$ ligase; $\mathrm{CHS}$ : Chalcone synthase; $\mathrm{CHI}$ : Chalcone isomerase; $\mathrm{F} 3 \mathrm{H}$ : Flavanone 3-hydroxylase; F35H: Flavonoid 3,5 -hydroxylase; DFR: Dihydroflavonols 4-reductase; ANS: Anthocyanin synthase; UFGT: UDP-glucose: Flavonoid 3-glucosyltransferase; FNS: Flavone synthase; FLS: Flavcnol synthase; MT: Metallothionein-like protein. SS: seedling stage; IE: Initial expansion; FE: Fullexpansion; BS: Bolting stage; IFS: Initial flowering stage; FBS: Full-bloom stage; PS: Podding stage; DEGs: Differential expression genes; KEGG: Kyoto encyclopedia of genes and genomes; FPKM: Fragments per kilobase of transcript per million mapped reads; GO: Gene ontology; NCBI: National center for biotechnology information; BP: Biological process; MF: Molecular function; CC: Cellular component.

\section{Declarations}

\section{Ethics approval and consent to participate}

Not applicable.

\section{Consent for publication}

Not applicable.

\section{Availability of data and materials}

All data generated or analysed during this study are included in this published article [and its supplementary information files].

\section{Competing interests}

The authors declare that they have no competing interest.

\section{Funding}

This work was supported by Natural Science Foundation of CSTB (cstc2019jcyj-msxm1573), Science and Technology Plan Projects of Fuling District (FLKJ, 2018BBB3009).

\section{Authors' Contributions}

Conceptualization, Jian Gao and Wenbo Li; Methodology, Mao Luo and Jian Gao; Software, Jian Gao and Mao Luo; Formal Analysis, Wen-Bo Li and Jian Gao; Resources, Hua Peng; Writing-Original Draft 
Preparation, Jian Gao; Writing-Review \& Editing, Jian Gao and Mao Luo; Supervision, WenBo Li; Funding Acquisition, Wenbo Li. All authors have read and approved the manuscript for publication.

\section{Acknowledgment}

Not applicable.

\section{References}

1. Khoo HE, Azlan A, Tang ST, Lim SM: Anthocyanidins and anthocyanins: colored pigments as food, pharmaceutical ingredients, and the potential health benefits. Food \& Nutrition Research 2017, 61(1):1361779.

2. Yousuf B, Gul K, Wani AA, Singh P: Health Benefits of Anthocyanins and Their Encapsulation for Potential Use in Food Systems: A Review. Crit Rev Food Sci Nutr 2015, 56(13):2223-2230.

3. He J, Giusti MM: Anthocyanins: Natural Colorants with Health-Promoting Properties. Annual Review of Food Science \& Technology 2010, 1(1):163.

4. Anju Bajpai, Kasim Khan, M. Muthukumar, S. Rajan, Singh NK: Molecular analysis of anthocyanin biosynthesis pathway genes and their differential expression in mango peel. Genome 2017, 61(3):157.

5. Aza-González C, Herrera-Isidrón L, Núñez-Palenius HG, Vega OMDL, Ochoa-Alejo N: Anthocyanin accumulation and expression analysis of biosynthesis-related genes during chili pepper fruit development. Biologia Plantarum 2012, 57(1):49-55.

6. Dao TTH, Linthorst HJM, Verpoorte R: Chalcone synthase and its functions in plant resistance. Phytochemistry Reviews 2011, 10(3):397-412.

7. Dewey CN, Li B: RSEM: accurate transcript quantification from RNA-Seq data with or without a reference genome. Bmc Bioinformatics 2011, 12(1):323-323.

8. Tarazona S, García F, Ferrer A, Dopazo J, Conesa A: NOlseq: a RNA-seq differential expression method robust for sequencing depth biases. University of Southampton 2012, 17(B):18.

9. Xie C, Mao X, Huang J, Ding Y, Wu J, Dong S, Kong L, Gao G, Li C-Y, Wei L: KOBAS 2.0: a web server for annotation and identification of enriched pathways and diseases. Nucleic Acids Research 2011, 39(Web Server issue):316-322.

10. Jian G, Mao L, Zhang C, Hua P, Lin H, Shen Y, Zhao M, Pan G, Zhang Z: A putative pathogen-resistant regulatory pathway between MicroRNAs and candidate target genes in maize. Journal of Plant Biology 2015, 58(4):211-219.

11. Schefe JH, Lehmann KE, Buschmann IR, Unger T, Funke-Kaiser H: Quantitative real-time RT-PCR data analysis: current concepts and the novel "gene expression'sCTdifference" formula. Journal of Molecular Medicine 2006, 84(11):901-910.

12. Ferreira D, Slade D, Marais JPJ: Flavans and Proanthocyanidins. 2005. 
13. GROTEWOLD E: The genetics and biochemistry of floral pigments. Annual Review of Plant Biology 2006, 57(1):761-780.

14. Xie, D.-Y.: Role of Anthocyanidin Reductase, Encoded by BANYULS in Plant Flavonoid Biosynthesis. Science 2003, 299(5605):396-399.

15. Cutanda-Perez MC, Ageorges A, Gomez C, Vialet S, Terrier N, Romieu C, Torregrosa L: Ectopic expression of VImybA1 in grapevine activates a narrow set of genes involved in anthocyanin synthesis and transport. Plant Molecular Biology 2009, 69(6):633.

16. Griesser M, Hoffmann T, Bellido ML, Rosati C, Fink B, Kurtzer R, Aharoni A, Munoz-Blanco J, Schwab W: Redirection of Flavonoid Biosynthesis through the Down-Regulation of an Anthocyanidin Glucosyltransferase in Ripening Strawberry Fruit. Plant Physiology 2008, 146(4):1528-1539.

17. Zhao J, Dixon RA: The 'ins' and 'outs' of flavonoid transport. Trends in Plant Science 2010, 15(2):7280.

18. Marrs KA, Alfenito MR, Lloyd AM, Walbot V: A Glutathione-S-Transferase Involved in Vacuolar Transfer Encoded by the Maize Gene Bronze-2. Nature 1995, 375(6530):397-400.

19. Kitamura S, Shikazono N, Tanaka A: TRANSPARENT TESTA 19 is involved in the accumulation of both anthocyanins and proanthocyanidins in Arabidopsis. Plant Journal 2010, 37(1):104-114.

20. Espley RV, Brendolise C, Chagne D, Kutty-Amma S, Green S, Volz R, Putterill J, Schouten HJ, Gardiner SE, Hellens RP: Multiple Repeats of a Promoter Segment Causes Transcription Factor Autoregulation in Red Apples. Plant Cell 2009, 21(1):168-183.

21. Xu W, Dubos C, Lepiniec Lc: Transcriptional control of flavonoid biosynthesis by MYB-bHLH-WDR complexes. Trends in Plant Science 2015, 20(3):176-185.

\section{Tables}

Table 1. Summary of raw reads for 14 samples (two replicates) of fleshy roots of seven growth phases 


\begin{tabular}{lcccc}
\hline Sample & Total_Read_Count & rRNA_Read_Count & Clean_Read_Count & Clean_Rate \\
\hline FBS_root_1 & 33990858 & 7871020 & 26119838 & $76.84 \%$ \\
\hline FBS_root_2 & 33990858 & 8386243 & 25604615 & $75.33 \%$ \\
\hline BS_root_1 & 33401149 & 7702113 & 25699036 & $76.94 \%$ \\
\hline BS_root_2 & 33401149 & 7587241 & 25813908 & $77.28 \%$ \\
\hline IE_root_1 & 28574219 & 4463319 & 24110900 & $84.38 \%$ \\
\hline IE_root_2 & 28574219 & 4663468 & 23910751 & $83.68 \%$ \\
\hline SS_root_1 & 20191382 & 504415 & 19686967 & $97.50 \%$ \\
\hline SS_root_2 & 20191382 & 560164 & 19631218 & $97.23 \%$ \\
\hline IFS_root_1 & 24547530 & 5534772 & 19012758 & $77.45 \%$ \\
\hline IFS_root_2 & 24547530 & 5976321 & 18571209 & $75.65 \%$ \\
\hline FE_root_1 & 14316434 & 3200500 & 11115934 & $77.94 \%$ \\
\hline FE_root_2 & 14316434 & 3092331 & 11224103 & $78.40 \%$ \\
\hline PS_root_1 & 20673951 & 6219688 & 14454263 & $70.08 \%$ \\
\hline PS_root_2 & 20673951 & 5789807 & 14884144 & $72.01 \%$ \\
\hline
\end{tabular}

\section{Figures}


Cluster 1

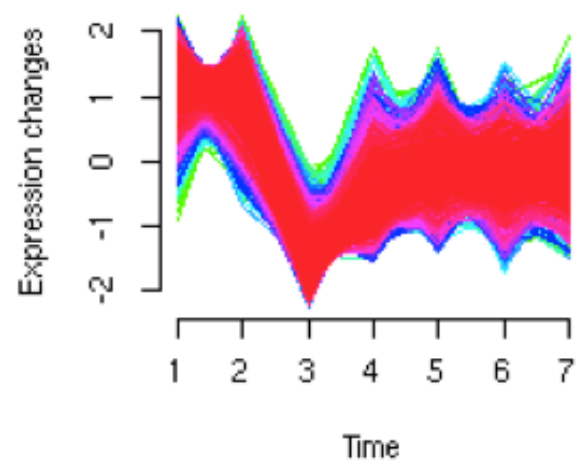

Cluster 4

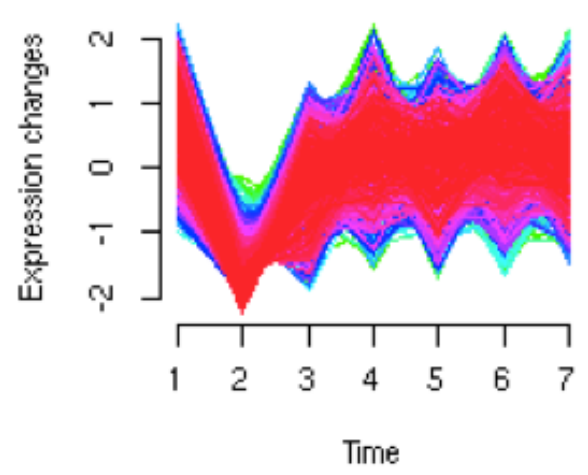

Cluster 7

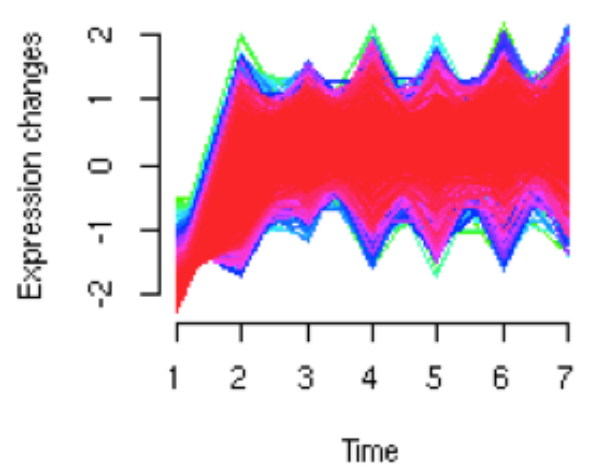

Cluster 2

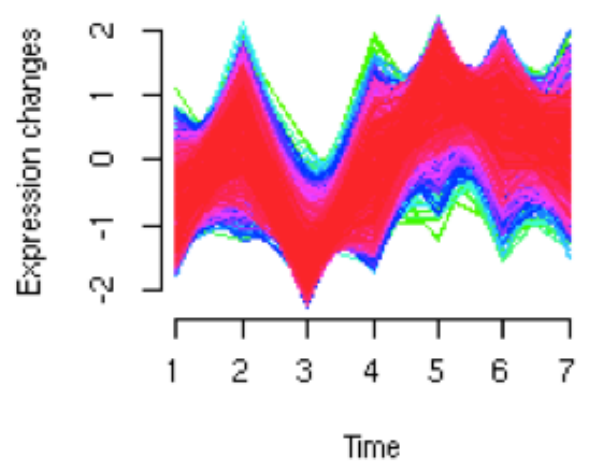

Cluster 5

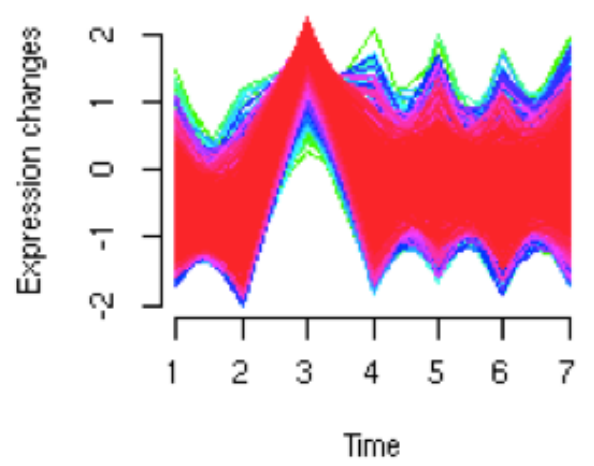

Cluster 8

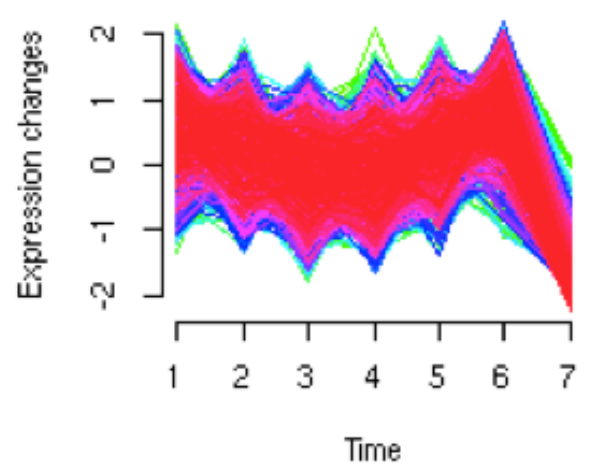

Cluster 3

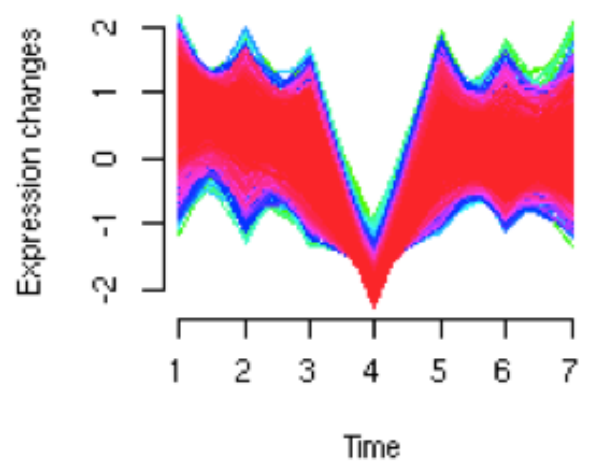

Cluster 6

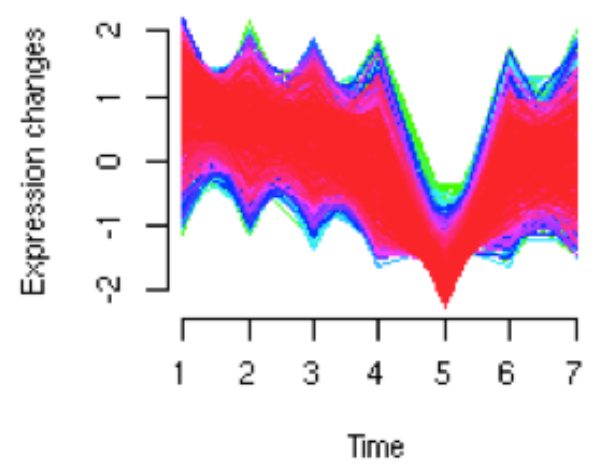

Cluster 9

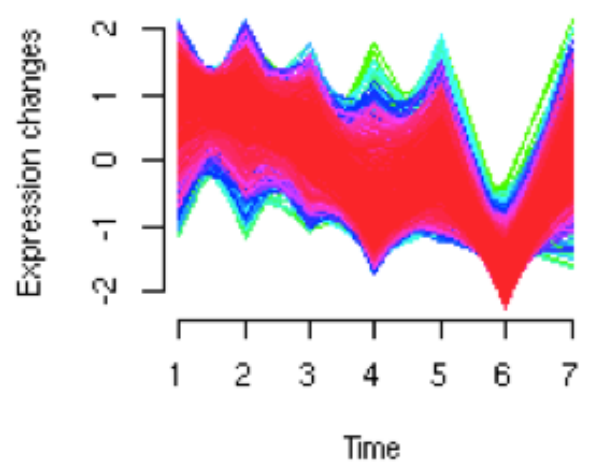

Figure 1

Normalized expression levels for all global expressed genes involved in the dynamics growing stages of fleshy roots in carmine radish. 
A

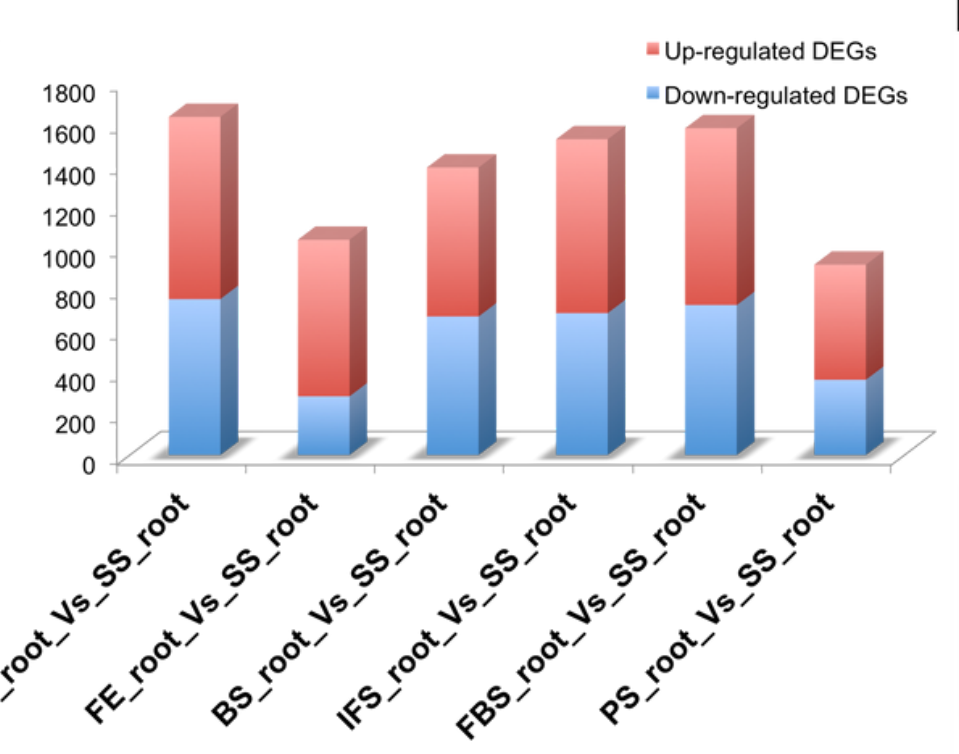

B

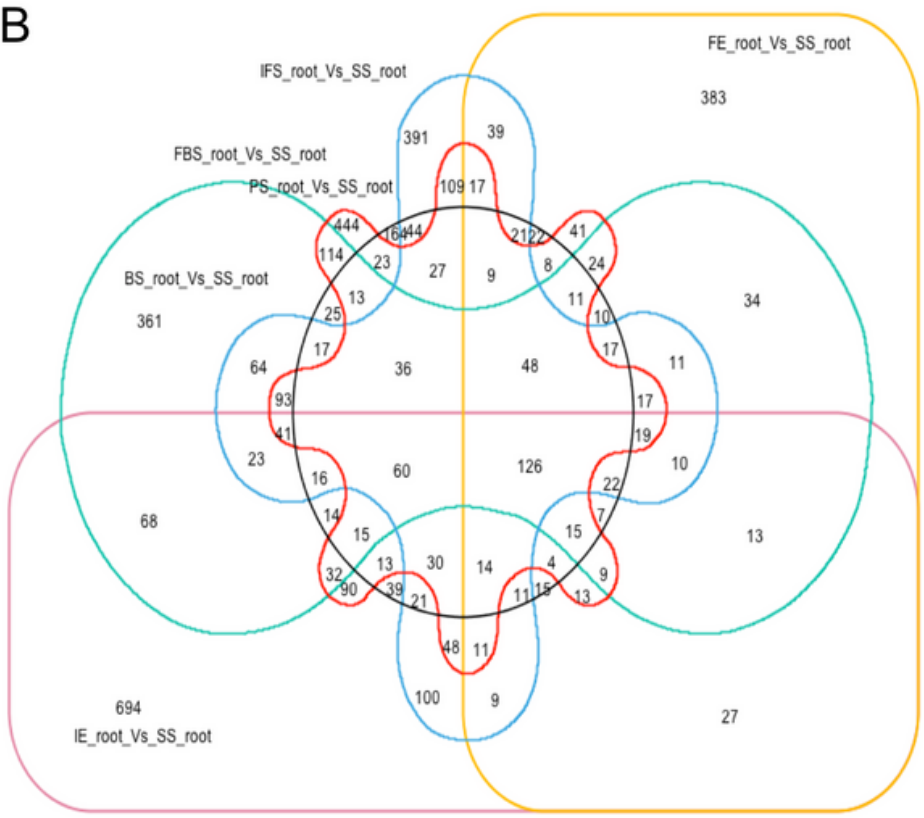

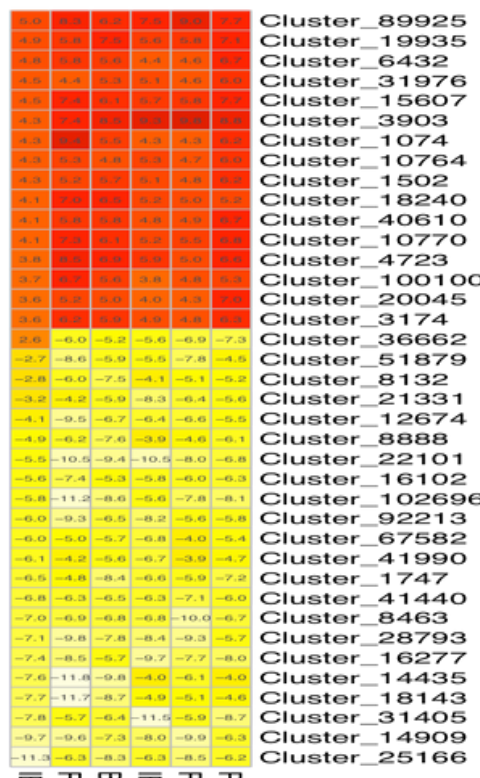

䎡而思贾品

흘 흐을

का का का

'心'心

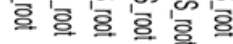

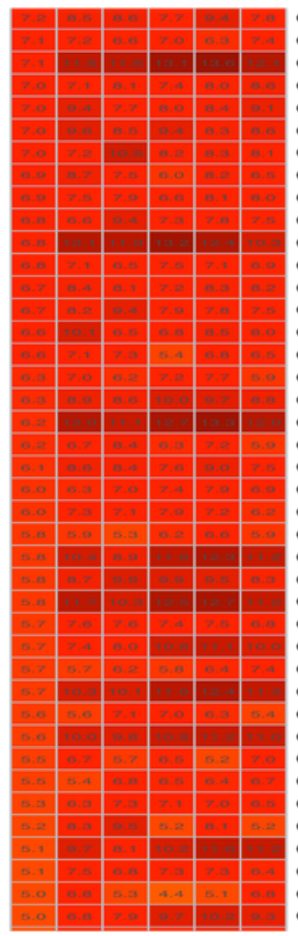

Cluster_46036 Cluster_25236 Cluster_13775 Cluster_30540 Cluster_29444 Cluster_13909 Cluster_27773 Cluster_15428 Cluster_6627 Cluster_35785 Cluster_38335 Cluster_115066 Cluster_25088 Cluster_2666 Cluster_2378 Cluster_63426 Cluster_30537 Cluster_35737 Cluster_28614 Cluster_15281 Cluster_56695 Cluster_4431 Cluster 20704 Cluster-102822 Cluster-7429 Cluster-1765 Cluster-29200 Cluster_29200 Cluster_39858 Cluster_14857 Cluster_113548 Cluster_113 Cluster_13830 Cluster_23793 Cluster-72625 Cluster_46678

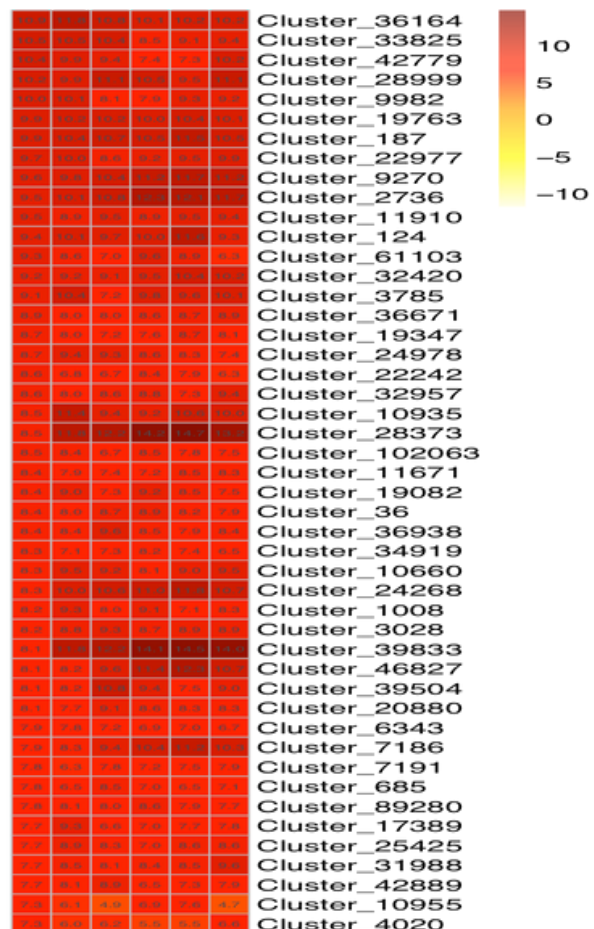

\section{Figure 2}

Transcriptional changes of DEGs involved in the dynamics growing stages of fleshy roots in carmine radish. A. Statistic of differentially expression genes (including up-regulated and down-regulated in each comparison groups) in the dynamics growing stages of fleshy roots ('IE_root', 'FE_root', 'BS_root', 'IFS_root', 'FBS_root' and 'PS_root'), compared with 'SS_root' group. B. Venny graph of co-modulated DEGs (Common DEGs in the dynamic growing stages of fleshyroot in carmine radish). C. Clustering and heat map of common differentially expressed (Co-modulated genes) based on the expression profiles in the dynamics growing stages of fleshy roots ('IE_root', 'FE_root', 'BS_root', 'IFS_root', 'FBS_root' and 'PS_root'), compared with 'SS_root' group. 


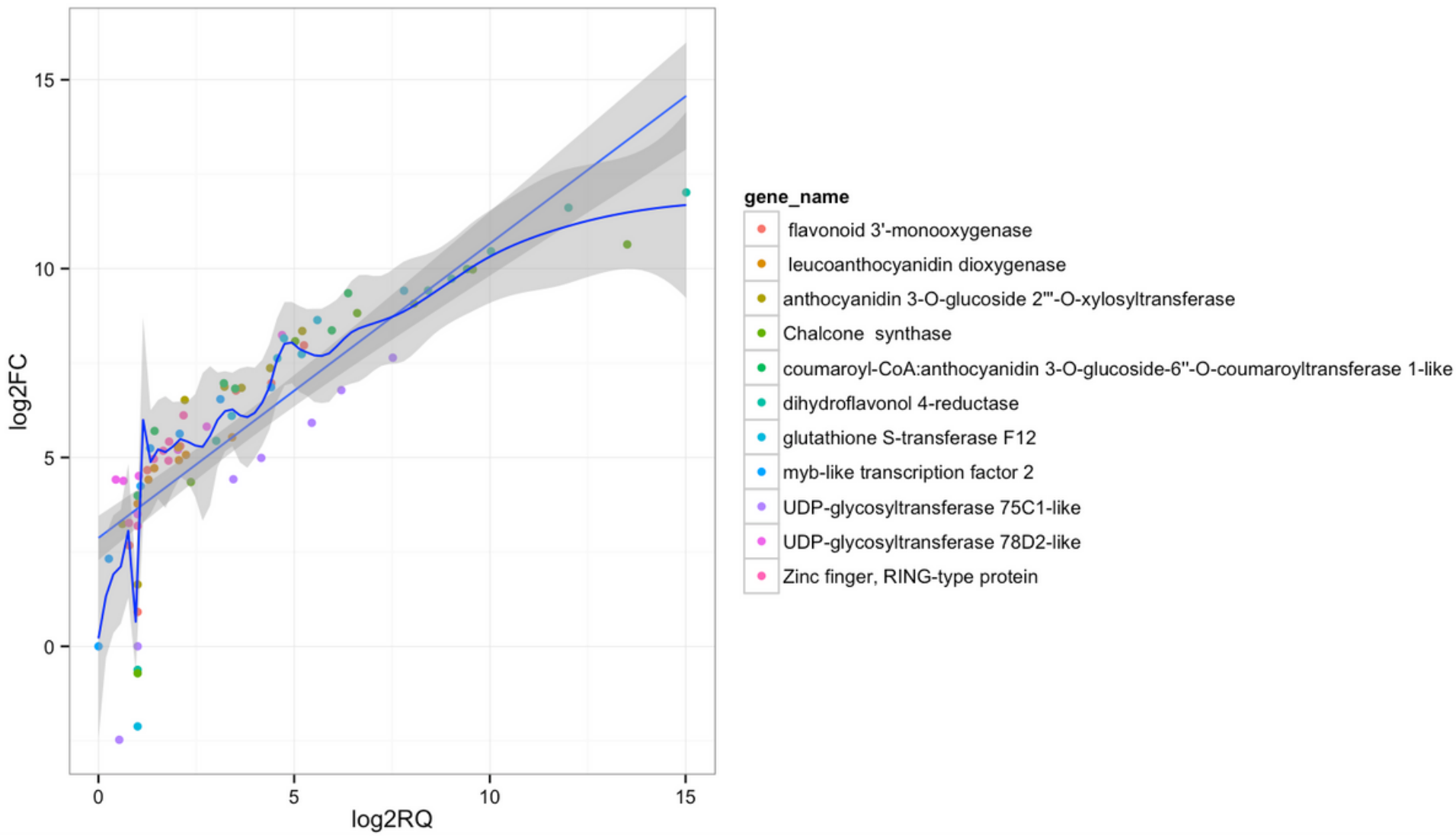

\section{Figure 3}

Validate of candidate Co-modulated DEGs involved in the dynamics growing stages of fleshy roots in carmine radish using qRT-PCR and then correlation between RNA-seq and qPCR data were conducted. Each RNA-seq expression data was plotted against that from quantitative real-time PCR and fit into a linear regression. Both $\mathrm{x}$ - and $\mathrm{y}$-axes were shown in $\log 2$ scale and each color represented a different gene. 


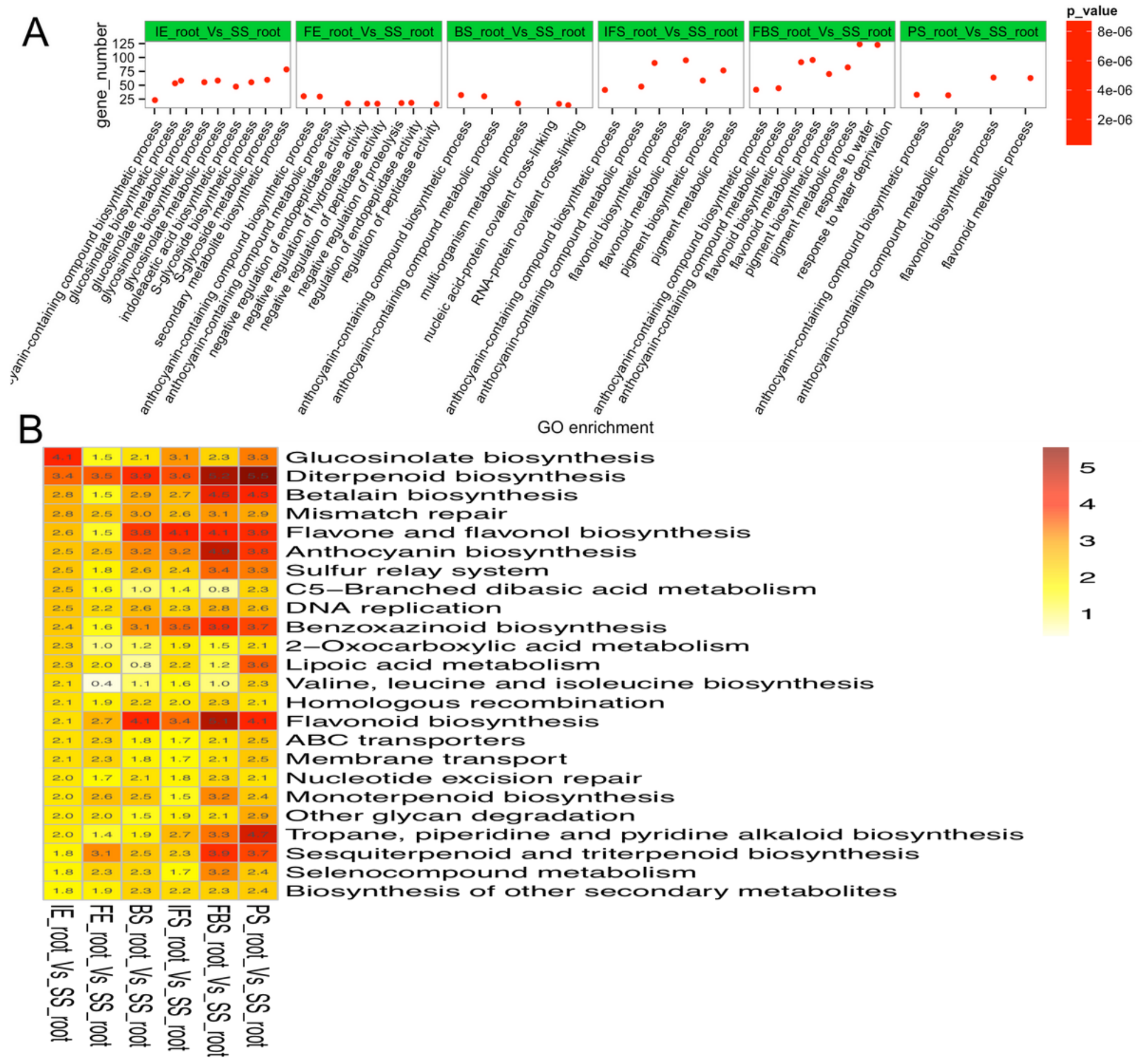

Figure 4

Functional enrichment analysis of differentially expressed genes (DEGs) related to the dynamics growing stages of fleshy roots in carmine radish. A. Enriched GO terms of DEGs related to the dynamics growing stages of fleshy roots in radish. GO terms are plotted on the ordinate, and the enrichment factor (rich factor) is plotted on the abscissa. The colour of points represents the q-value, and the size of points represents the number of DEGs mapped to the reference pathway. Legends for the colour scale of qvalues and size-scaling of the number of DEGs are shown to the right of the plot. B. Pathway enrichment analysis among differentially expressed genes related to anthocyanin synthesis in radish. Enriched KEGG 
pathway terms divided by the dynamics growing stages(IE_root', 'FE_root', 'BS_root', 'IFS_root', 'FBS_root' and 'PS_root'), compared with 'SS_root'. Red color indicates statically overrepresented.

\section{Supplementary Files}

This is a list of supplementary files associated with this preprint. Click to download.

- supplement1.png

- supplement2.png

- supplement3.xIsx 